\title{
Genetic Variability and Sequence Relatedness of Matrix Protein in Viruses of the Families Paramyxoviridae and Pneumoviridae
}

\author{
Anamarija Slovic ${ }^{a, b}$ Tanja Kosutic-Gulija ${ }^{a, b}$ Maja Santak ${ }^{a, b}$ \\ Jelena Ivancic-Jeleckia, b Maja Jagusic ${ }^{a, b}$ Sunčanica Ljubin-Sternak ${ }^{c, d}$

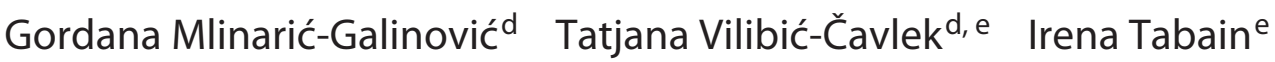 \\ Dubravko Forcic ${ }^{a, b}$ \\ ${ }^{a}$ Centre for Research and Knowledge Transfer in Biotechnology, University of Zagreb, Zagreb, Croatia; \\ ${ }^{b}$ Center of Excellence for Viral Immunology and Vaccines, CERVirVac, Zagreb, Croatia; ${ }^{\circ}$ Teaching Institute of \\ Public Health "Dr. Andrija Štampar", Zagreb, Croatia; 'School of Medicine, University of Zagreb, Zagreb, Croatia; \\ ${ }^{\text {e}}$ Croatian National Institute of Public Health, Zagreb, Croatia
}

\section{Keywords}

Paramyxoviruses · Pneumoviruses · Matrix protein ·

Genetic variability

\begin{abstract}
Background: The families Paramyxoviridae and Pneumoviridae comprise a broad spectrum of viral pathogens that affect human health. The matrix $(M)$ protein of these viruses has a central role in their life cycle. In line with this, molecular characteristics of the $M$ proteins from variable viruses that circulated in Croatia were investigated. Methods: Sequences of the M proteins of human parainfluenza virus (HPIV) 1-3 within the family Paramyxoviridae, human metapneumovirus (HMPV), and human respiratory syncytial virus from the family Pneumoviridae were obtained and analyzed. Results: M proteins were very diverse among HPIVs, but highly conserved within each virus. More variability was seen in nucleotide sequences of $M$ proteins from the Pneumoviridae family. An insertion of 8 nucleotides in the $3^{\prime}$ untranslated region in 1 HMPV M gene sequence was discovered (HR347-12). As
\end{abstract}

\section{KARGER}

(c) 2018 S. Karger AG, Basel

E-Mail karger@karger.com

www.karger.com/int there are no samples with such an insertion in the database, this insertion is of interest and requires further research. Conclusion: While we have confirmed that M proteins were conserved among individual viruses, any changes that are observed should be given attention and further researched. Of special interest is inclusion of HPIV2 M proteins in this analysis, as these proteins have not been studied to the same extent as other paramyxoviruses.

(c) 2018 S. Karger AG, Basel

\section{Introduction}

The families Paramyxoviridae and Pneumoviridae, within the Mononegavirales order, comprise a broad spectrum of viral pathogens, many of which affect human health. Important human pathogens within the Paramyxoviridae family include mumps, measles, and human parainfluenza virus 1-4 (HPIV 1-4), as well as the deadly Hendra and Nipah viruses, whereas human respiratory syncytial virus (HRSV) and human metapneumovirus 
(HMPV) belong to the Pneumoviridae family [1]. All of these viruses have a nonsegmented, negative sense singlestranded RNA genome and share 6 genes: nucleoprotein $(\mathrm{N} / \mathrm{NP})$, phosphoprotein $(\mathrm{P})$, matrix protein $(\mathrm{M})$, fusion glycoprotein (F), attachment glycoprotein [hemagglutinin-neuraminidase $(\mathrm{HN})$, hemagglutinin $(\mathrm{H})$, or glycoprotein $(G)$ ], and large RNA-dependent RNA polymerase (L) [2]. In 2017, non-Latinized binomial species names replaced all paramyxovirus and pneumovirus species names [3]. It is important not to confuse a virus species (which is a taxonomic concept that does not have a sequence and cannot be isolated, transmitted to a host, or otherwise manipulated) with a virus (a physical entity that can be isolated and manipulated experimentally) [4]. Throughout this report, we use terms HPIV1, HPIV3, HPIV2, HRSV, and HMPV for viruses belonging to the species Human respirovirus 1 , Human respirovirus $3, \mathrm{Hu}$ man rubulavirus 2, Human orthopneumovirus, and $\mathrm{Hu}$ man metapneumovirus, respectively.

$\mathrm{M}$ protein coordinates the assembly of new virions by interacting both with cytoplasmic tails of viral glycoproteins and viral ribonucleoprotein complex (RNA genome bound with $\mathrm{N}$ or NP protein), concentrating viral components on specific sites at the membrane from which viruses bud [5]. Interaction with viral glycoproteins was demonstrated by the loss of colocalization when cytoplasmic tails of $\mathrm{HN}$ protein, or both $\mathrm{HN}$ and $\mathrm{F}$ protein of parainfluenza virus 5 were truncated $[6,7]$. The same was shown for measles virus and HRSV $[8,9]$, indicating the importance of these interactions for particle production. The region important for the interaction of $\mathrm{M}$ protein with nucleocapsid has been demonstrated for measles virus, where it was shown that the region close to the Cterminal end of $\mathrm{N}$ protein is indispensable for these interactions [10]. The same was demonstrated for C-terminal regions of parainfluenza 5 and Nipah virus $\mathrm{N}$ proteins containing the DLD sequence [11].

The central role of M protein during infection has been shown in experiments where mutations of the M protein sequence led to impaired viral production $[12,13]$, with almost complete loss of particle production when the $M$ gene is absent $[14,15]$. Also, it has been shown that for many of these viruses production of virus-like particles (VLP) from transfected cells depends solely on the expression of M protein, including HPIV1 [16], Sendai virus [17], and measles virus [18], although for some viruses coexpression with other viral proteins leads to more efficient VLP production [19, 20].

The aim of this study was to investigate molecular characteristics of the $\mathrm{M}$ proteins from highly variable
HPIV1-3, HMPV, and HRSV viruses that circulated in Zagreb, Croatia, and to compare these changes with the observed changes in other viral genes. Sequences were collected over a time period of 6 years (2011-2016) for HPIV1, HPIV3, and HMPV virus, 5 years for HPIV2 (2011-2015), and 8 years for HRSV (2006-2008, 20112015).

\section{Materials and Methods}

\section{Clinical Samples}

The clinical samples included nasopharyngeal secretions obtained from hospitalized patients presenting with acute respiratory infection, collected in the Zagreb region. Samples were chosen based on their previously determined sequences of major glycoprotein genes (HRSV [21, 22], HPIV1 [23], HPIV2 [24], HPIV3 [25], HMPV [26]) such that different viruses that have been observed throughout the study period were included.

The study was approved by the Ethics Committees of the Croatian National Institute of Public Health, University Children's Hospital Zagreb, and University Hospital for Infectious Diseases and Medical School of the University of Zagreb.

\section{Reverse Transcription, PCR, and Sequencing}

Total RNA was isolated from $500 \mu \mathrm{L}$ of clinical samples or from $250 \mu \mathrm{L}$ of Vero cell supernatants according to the method of Chomczynski and Sacchi [27]. The cDNA was synthesized using random hexamers and MuLV reverse transcriptase (Thermo Fisher Scientific, Waltham, MA, USA) and the synthesis was carried out at $42^{\circ} \mathrm{C}$ for $60 \mathrm{~min}$, followed by $95^{\circ} \mathrm{C}$ for $5 \mathrm{~min}$. The primer sets used to amplify and sequence the full-length $\mathrm{M}$ gene for every virus as well as detailed PCR procedure are shown in online supplementary Table 1 (see www.karger.com/doi/10.1159/000487049 for all online suppl. material) and the corresponding text. The PCR products were gel purified with QIAQuick Gel Extraction Kit (Qiagen, Hilden, Germany). Sequencing reactions were set up with purified DNA, primers specific for M gene (online suppl. Table 1), and the BigDye Terminator v.3.1 Cycle Sequencing Kit (ThermoFisher Scientific, Waltham, MA, USA), according to the protocol recommended by the manufacturer. Sequencing and sequence analysis was performed on a 3130 Genetic Analyser (Thermo Fisher Scientific, Waltham, MA, USA).

\section{Multiple Sequence Alignments and Phylogenetic Analysis}

Nucleotide and amino acid sequences were aligned using the MAFFT multiple sequence alignment tool available at the EMBLEBI website (http://www.ebi.ac.uk/tools/msa/mafft), and edited in BioEdit v.7.2.5. Sequence conservation (defined as the percentage of genomic positions identical in all strains; gaps were ignored during computing) was calculated using MEGA6 (Molecular Evolutionary Genetics Analysis) software [28]. The proportion of nonsynonymous $(\mathrm{dN})$ and synonymous sites $(\mathrm{dS})$ was calculated with the single-likelihood ancestor counting (SLAC), FEL (fixed effects likelihood), and MEME (mixed effects model of evolution) method available on the Datamonkey server (http://www.datamonkey. org/) [23]. Phylogenetic trees were generated using the maximum likelihood method with MEGA6 software [28], under the most ap- 


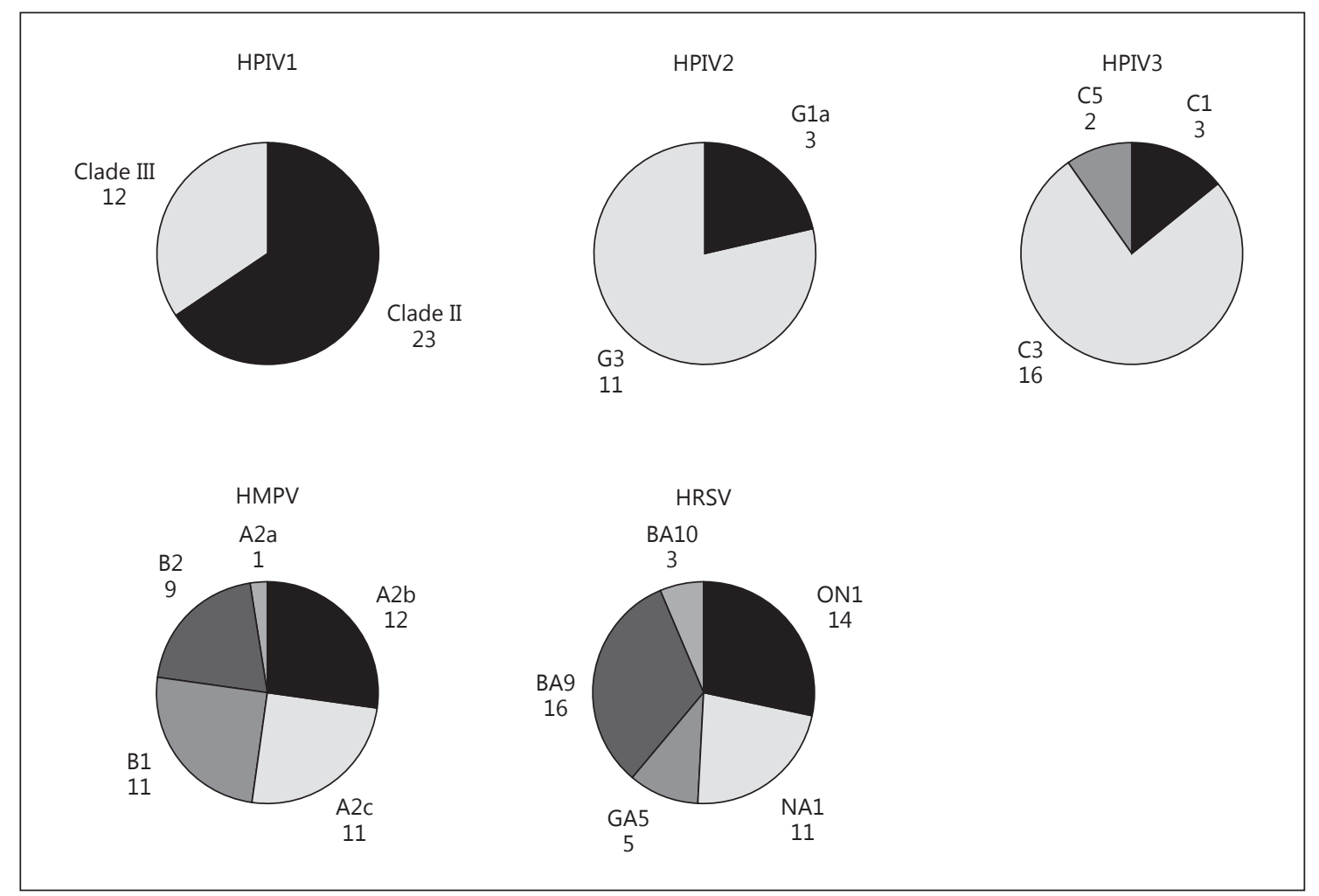

Fig. 1. The number of strains in each genotype or clade included in this study.

propriate model of nucleotide (nt) substitution determined with jModeltest v.2.1.4 [24].

The unique $\mathrm{M}$ gene sequences obtained in this study were submitted to GenBank under accession numbers: MF166637 to MF166662 for HPIV1, MF133335 to MF133348 for HPIV2, MF166670 to MF166674 for HPIV3, MF104588 to MF104624 for HMPV, and MF104625 to MF104671 for HRSV. A list of accession numbers of corresponding glycoprotein sequences is given in online supplementary Table 2 .

\section{Analysis of Deduced Amino Acids}

Amino acid variability was determined by calculating the Shannon entropy implemented in BioEdit software. A Shannon entropy plot was generated by exporting and plotting calculated entropy values against their corresponding amino acid positions in the alignment. Kyte-Doolittle hydropathy profiles were generated using ExPASy Protscale tool (http://web.expasy.org/protscale) and a window size of 9 .

\section{Results}

\section{Selection of HPIV Samples for M Gene Analysis}

The 4 serotypes of HPIV are distributed among 2 different genera (Respirovirus and Rubulavirus) within the Paramyxoviridae family, indicating their high diver- gence, which is also demonstrated by low amino acid sequence identity of $\mathrm{F}$ and $\mathrm{HN}$ proteins between these viruses [25].

For the purpose of this study, we analyzed 35 complete M gene sequences belonging to HPIV1, 14 sequences that belong to HPIV2, and 21 sequences from HPIV3. Different viruses representing all genotypes and clades observed in the phylogenetic tree constructed with either the F or $\mathrm{HN}$ gene were included. The number of strains in each genotype and clade included in this study is shown in Figure 1, while the supporting phylogenetic tree of HPIV M genes is shown in online supplementary Figure 1a.

\section{Variability among M Proteins of HPIVs}

Out of 35 HPIV1 M gene sequences, there were 28 unique sequences considering the coding region, with 11 of them having a unique protein sequence (online suppl. Table 3). Despite the lower number of HPIV2 samples (14), and high percentage of both nucleotide and amino acid identity (Table 1), there were no samples that had identical nucleotide sequences, and 8 of the samples had a unique $M$ protein sequence (Fig. 2; online suppl. Table $3)$. The third serotype had a somewhat lower percentage 
Fig. 2. Deduced amino acid alignment of $M$ protein from Croatian HPIV strains shown relative to the sequence of strain HPIV1s/ Zagreb.HR/3.12(1020). For clarification, HPIV2 sequences have been realigned relative to HPIV2i/Zagreb.HR/47.15(1779) and shaded in darker gray, while HPIV3 sequences are shown relative to HPIV $3 \mathrm{~s} / \mathrm{Za}$ greb.HR/10.11(6622) and shaded in lighter gray. Residues conserved within all 3 serotypes are denoted with black dots below the alignment. The genotype assignment is shown on the right. Identical residues are indicated by small dots, and dashes designate gaps corresponding to nucleotide insertions.
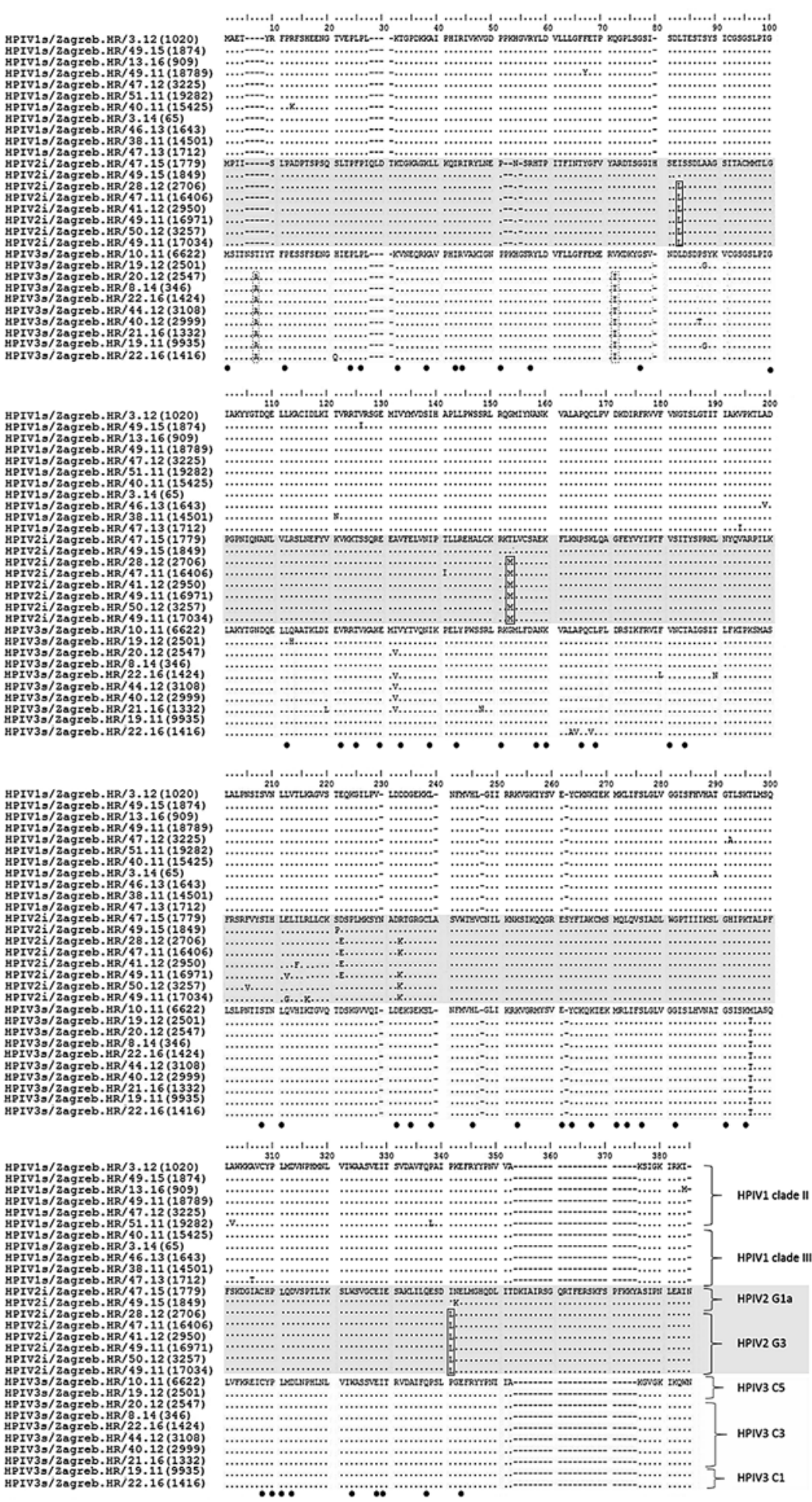
Table 1. M gene nucleotide and amino acid identity percentages of paramyxovirus and pneumovirus strains included in this study

\begin{tabular}{llll}
\hline Virus & $\begin{array}{l}\text { Nucleotide } \\
\text { identity, } \%\end{array}$ & $\begin{array}{l}\text { Amino acid } \\
\text { identity, } \%\end{array}$ & $\mathrm{dN} / \mathrm{dS}$ \\
\hline HPIV1 & 90.1 & 96.5 & 0.047 \\
HPIV2 & 90.5 & 96.8 & 0.084 \\
HPIV3 & 86.8 & 95.7 & 0.069 \\
HMPV & 73.9 & 96.4 & 0.011 \\
HRSV & 72.2 & 91 & 0.05 \\
\hline
\end{tabular}

of nucleotide identity (Table 1), with 20 out of 21 samples having a unique nucleotide sequence, resulting in 10 different $M$ protein sequences (Fig. 2; online suppl. Table 3). Observed amino acid differences could be associated with different subgroups in the case of HPIV2 and HPIV3 (Fig. 2); no pattern was present within the HPIV1 sequences. The mean $\mathrm{dN} / \mathrm{dS}$ values, calculated by the SLAC method, for all HPIV serotypes were very low: 0.047, 0.084, and 0.069 for HPIV1, HPIV2, and HPIV3, respectively, indicating that substitutions are unfavored and purified from the population. Similar results were obtained when the MEME and FEL methods were implemented (data not shown). The majority of changes that were observed for each studied serotype were conservative, confirming the rigid nature of changes that are allowed within the M protein.

The calculated nucleotide and amino acid sequence identity shows a high percentage of conservation for all 3 serotypes individually (Table 1), but the percentage of identity among the 3 serotypes was very low (15\%); only 52 positions were completely conserved (Fig. 2). These positions were randomly distributed along the alignment. HPIV 1 and HPIV 3 share a much higher percentage identity between their M proteins (60\%), while this percentage drops to $22 \%$ when each of these 2 serotypes is compared with HPIV2.

\section{Analysis of HPIV M Proteins}

Among HPIV serotypes, HPIV2 had the longest M protein (377 amino acids), which was 24 or 29 amino acids longer than the M protein of HPIV3 or HPIV1, respectively (Fig. 2). Kyte-Doolittle hydropathy profiles were generated for each serotype, based on their consensus protein sequence, to reduce noise that would arise by including all sequences. All 3 serotypes exhibited a similar pattern, with numerous hydrophobic residues and continuous hydrophilic areas; the exception was the afore-

Analysis of Matrix Proteins in Families

Paramyxoviridae and Pneumoviridae mentioned part of $\mathrm{M}$ protein present only in the HPIV2 sequences, which contains hydrophilic residues in the Cterminal end (online suppl. Fig. 2a).

In order to search for conserved regions, variability among aligned $\mathrm{M}$ protein sequences at each position was calculated using Shannon entropy. We eliminated positions that contain gaps, because gaps would be considered as another character state and would give higher entropy values. The range of detected entropy values was from 0 to 1.137 , with the highest entropy value calculated for position 72, where 4 different amino acids are present (Fig. 2). In confirmation with the calculated high variability among $\mathrm{M}$ proteins of the 3 serotypes, a minority of residues showed a low degree of heterogeneity, whereas many residues had entropy scores above 1 (91 out of 385 positions).

\section{Selection HRSV and HMPV Samples for M Gene Analysis}

HRSV and HMPV are important human pathogens within the family Pneumoviridae (previously assigned as a subfamily in the Paramyxoviridae family), which are characterized by fast evolution followed by the exchange of predominant genotypes [29-32]. Forty-four complete HMPV M gene sequences were included in the analysis. Twenty-four samples belonged to genotype A and 20 belonged to genotype B. Thirty HRSV group A and 19 group $B$ sequences were chosen for this study. The distribution of included HMPV and HRSV samples according to genotype is shown in Figure 1, with the supporting phylogenetic tree available in online supplementary Figure $1 \mathrm{~b}$.

\section{Variability among HMPV and HRSV M Proteins}

Matrix genes of HMPV showed more variability than HPIVs; 37 of 44 sequences had a unique sequence and the nucleotide identity among complete genes was only $73 \%$ (Table 1). When only coding regions were compared, this percentage slightly increased (77\%), but there were 36 unique sequences. This diversity was not reflected in the protein sequence; $M$ protein was highly conserved with a total of 5 different $M$ proteins (Fig. 3; online suppl. Table 3 ) and amino acid identities similar to those calculated for HPIVs (Table 1). Therefore, even though there are many observed substitutions in the nucleotide sequences, the majority of these are synonymous substitutions, with a very low mean $\mathrm{dN} / \mathrm{dS}(0.011)$ calculated with the SLAC method; this value was similar to $\mathrm{dN} / \mathrm{dS}$ values obtained with the MEME and FEL methods (data not shown). Nevertheless, these substitutions could distinguish between the 2 major genotypes, and there was 1 substitution that separated the A2c subgroup from the A2b subgroup (po- 
Fig. 3. Deduced amino acid alignment of $M$ proteins from Croatian HRSV and HMPV strains shown relative to the sequence of strain HRSV HR266-14. For clarification, HMPV sequences have been realigned relative to HMPV sequence HR1105-15. Residues conserved within both viruses are denoted with asterisks below the alignment. The genotype assignment is shown on the right. Identical residues are indicated by small dots, and dashes designate gaps corresponding to nucleotide insertions. Amino acid substitutions characteristic for the HRSV B group are indicated within closed rectangles, while substitutions that separate HMPV A and B group are denoted by arrows below the alignment. An amino acid substitution characteristic for the HMPV A2c subgroup is shaded in gray and the dashed rectangle marks an amino acid substitution characteristic for the HRSV BA9 genotype.

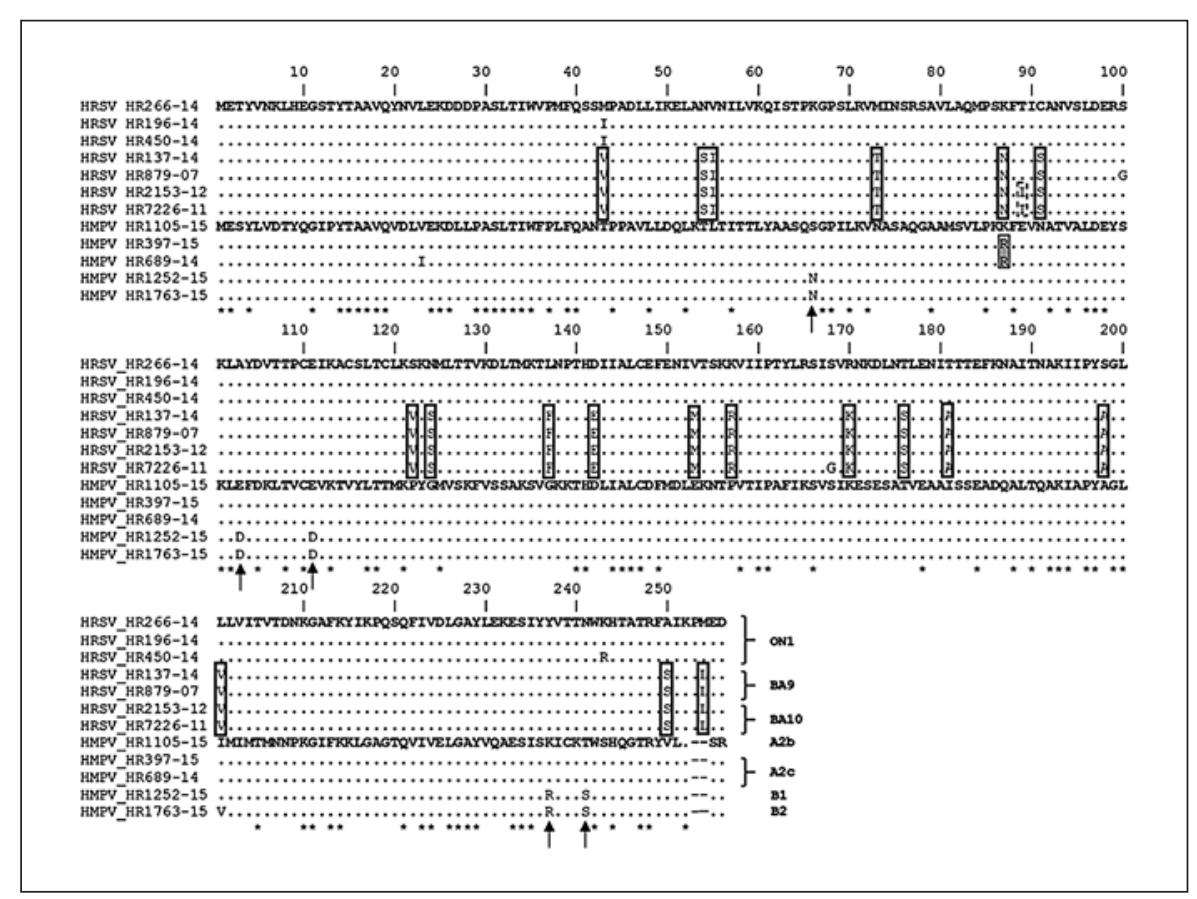

sition 87; Fig. 3). Since the only sample that belonged to the A2a subgroup (HR347-12) had an M protein sequence identical to HR1105-15, a representative of the A2b subgroup, we assume that these substitutions are specific for the A2c subgroup.

One genotype A sequence (HR347-12, acc. No. MF104612) had 8 nucleotides inserted in the $3^{\prime}$ untranslated region (in respect to all other genotype A sequences). Sample HR347-12 is the only sequence among samples included in this study that belongs to subgroup A2a. When we searched the GenBank database for similar sequences, there were no sequences with such an insertion in the $\mathrm{M}$ gene, and the closest match was a sequence isolated in Australia in 2003 (KC403981). This sequence belongs to the same subgroup, according to both the $\mathrm{M}$ and F gene phylogenetic tree, respectively (data not shown).

Similar results regarding variability among $M$ genes were obtained when the HRSV M gene sequences were analyzed, although different lengths of $\mathrm{M}$ genes are observed for 2 major groups (963 nt in HRSV A and 1,047 nt in HRSV B). A total of 47 unique sequences shared a nucleotide identity of $72 \%$ (Table 1 ), with more differences located toward the $3^{\prime}$ end. Again, the M protein was conserved with a dN/dS of 0.05 calculated with the SLAC method, which was similar to values calculated with the MEME and FEL methods (data not shown). Only 7 different M proteins were observed (online suppl. Table 3), which shared $91 \%$ identity (Table 1). Observed differences could distinguish between the major HRSV groups, and 1 amino acid residue separated the BA9 and BA10 strains (position 89; Fig. 3).

Within the Pneumoviridae family, M proteins showed 91 conserved positions (35.8\%). High variability between $\mathrm{M}$ proteins of these closely related viruses was shown by plotting the Shannon entropy. Again, many positions had high entropy scores, and these residues were spread along the complete alignment (not shown). The range of entropy scores was between 0 and 1.24, and the highest entropy was calculated for residue 43 where 4 amino acids are present (Fig. 3), which falls between a beta sheet and an alfa domain [21]. Similar properties of $M$ proteins within the Pneumoviridae family were shown when hydropathy profiles were generated based on consensus sequences of both viruses (online suppl. Fig. 2b).

\section{Discussion}

In spite of the fundamental role of $\mathrm{M}$ protein in the life cycle of paramyxoviruses and pneumoviruses, it is often overlooked as glycoproteins are the major focus of research of these viruses. It is especially interesting how $\mathrm{M}$ proteins of different viruses in these families have similar functions, even though they differ greatly both in length 
and amino acid sequence $[22,33]$, and how despite vast sequence divergence their tertiary structure is conserved [34]. Still, many of the observed interactions and mechanisms by which $M$ proteins regulate particle assembly remain poorly understood [5]. In line with the important function carried by this protein, we sought to investigate molecular characteristics of the M proteins from highly variable HPIV1-3, HMPV, and HRSV viruses. The time frame of included samples corresponds to some of the major shifts in genotype circulation, and thus the included viruses have undergone important evolutionary changes.

Firstly, we investigated versatility among the M proteins of HPIV1-3. As expected, M proteins were very diverse among 3 serotypes, even though they were highly conserved within each serotype. This is in line with amino acid identities among these serotypes calculated on the basis of both $\mathrm{F}$ and $\mathrm{HN}$ glycoproteins, where these glycoproteins share less than $50 \%$ sequence identity [25]. Similar to these results, M proteins of HPIV1 and HPIV3 were more closely related, with $60 \%$ amino acid identity between them, which corresponds to the identity between the L proteins of HPIV1 and HPIV3 [25]. However, most of the substitutions observed in the nucleotide sequences are synonymous, which was confirmed with very low $\mathrm{dN} / \mathrm{dS}$ values for each serotype, pointing out that $M$ protein function depends on the stability of its structure. Indeed, despite low amino acid identity among these proteins, similarities were observed regarding their overall hydropathy profiles, which is in agreement with the study by Latiff et al. [22].

Matrix proteins of HPIV1 and HPIV 3 are alone sufficient for the budding and formation of VLP $[16,35]$, indicating that the $\mathrm{M}$ protein of these viruses may be sufficient to induce membrane deformation leading to viral egress [36]. Critical residue for the formation of HPIV3 VLP has been mapped to leucine in the $\mathrm{C}$-terminal region of $\mathrm{M}$ protein (L302), a residue that is also indispensable for its ubiquitination [35], and this residue was completely conserved among our strains. Similar regions that are involved in VLP production have also been found for mumps virus (21-FPVI-24) [19], Nipah virus (62-YMYL65 and 92-YPLGVG-97) [37, 38], and Sendai virus (49YLDL-52) [39]. Such a sequence has not yet been determined for HPIV1, although there is a 50-YLDV-53 motif conserved within HPIV1 samples, which could be involved in the budding of HPIV1 by the analogy with the sequence of antigenically related Sendai virus.

Interestingly, in the alignment of HPIV2 and HPIV3 $\mathrm{M}$ proteins, some amino acid substitutions could be associated with different genetic clusters, while no such pattern was observed in HPIV1 (Fig. 2). This is especially interesting as no changes that could distinguish different phylogenetic subgroups were present when we analyzed HPIV3 $\mathrm{F}$ and HN proteins [40], which are more diverse, and therefore used for phylogenetic classification [41, 42]. Also, in the case of HPIV3, most of the amino acid changes in glycoproteins were restricted to cytoplasmic and transmembrane regions [40], which are regions thought to be important for interaction with the M proteins [43]. The fact that we could not observe any connection between substitutions and genetic clusters for HPIV1 samples might indicate that these groups contain viruses that are less divergent.

More variability was seen in nucleotide sequences of the Croatian M proteins from the Pneumoviridae family, which is expected since these viruses are also more diverse regarding their glycoprotein genes [26, 44]. Major HRSV $A$ and $B$ groups can be distinguished on the basis of different lengths of $M$ genes, a feature not present in other analyzed viruses. However, again the vast majority of changes were synonymous changes, thus HMPV and HRSV M proteins were very conserved, indicating that little protein variability is tolerated. Although these viruses are closely related, their $\mathrm{M}$ proteins shared a smaller number of completely conserved residues compared to the $\mathrm{M}$ proteins of HPIV1 and HPIV3, respectively. Nevertheless, their general properties were conserved, which is in line with common function and the architecture shared among $\mathrm{M}$ proteins, regardless of their primary sequences [45].

Residues important for HMPV budding have been mapped to a YAGL motif, highly conserved within pneumoviruses [46]. Within our sequences, this motif (residues 197-200) is conserved except in the case of 3 HRSV sequences (HR266-14, HR196-14, and HR468-14), which have serine in the place of alanine (Fig. 3); the role of this substitution was not examined.

In a recent study, Li et al. [47] showed that a multiepitope peptide, constructed by combining predicted B-cell, cytotoxic T lymphocyte (CTL), and T helper (Th) lymphocyte epitopes of HMPV can elicit both humoral immunity and cell-mediated immunity in mice. Since activated Th cells have role in CTL responses and memory [48], it is interesting that 1 of the 2 Th epitopes was predicted in M protein and is completely conserved in all our analyzed strains (position 36-50; Fig. 3).

Despite a conservative nature of $\mathrm{M}$ protein, there were still substitutions that could separate major genotypes in both viruses, and there was even 1 substitution in HMPV $M$ proteins that could distinguish a recently discovered A2c subgroup $[26,49]$ from the other 2 subgroups. This was unexpected, as such substitutions were previously 
only present in $\mathrm{G}$ proteins, for which extensive variations are probably the result of immunogenic pressure in a similar manner as was postulated for HRSV [50]. Such substitutions were not seen within $\mathrm{F}$ or $\mathrm{SH}$ glycoproteins [26], indicating the importance of extending research to other genes in cases when novel subgroups arise.

An insertion of 8 nucleotides was discovered in 1 HMPV Mgene sequence (HR347-12, acc. No. MF104612), which belongs to the A2a subgroup. These inserted nucleotides are located in the $3^{\prime}$ untranslated region, and there are no samples which have such insertion in the GenBank database. For paramyxoviruses and pneumoviruses, sequences at the ends of the genes are required for efficient termination of transcription [51-53] and noncoding regions in HMPV are mostly conserved [54], therefore this result was unexpected. In the case of HPIV3, Lingemann et al. [55] have shown that gene-end transcription signals of the M, NP, and P protein genes are identical, except for an 8-nucleotide insert present in the $M$ protein gene end. This insert was associated with an increased synthesis of read-through mRNA transcripts containing both the $\mathrm{M}$ and $\mathrm{F}$ gene, while removal of this insert shifted transcription to monocistronic $\mathrm{F}$ mRNA [55]. Therefore, observed insertion in the HMPV M gene is of interest and requires further research to determine the impact that this insertion has on the transcriptional level of $\mathrm{F}$ protein mRNA.

In this study, we analyzed $M$ proteins from highly variable viruses belonging to the 2 viral families, for which most research is concentrated on the analysis of viral glycoproteins. Of special interest is inclusion of HPIV2 $\mathrm{M}$ proteins in this analysis, as these proteins have not been studied to the same extent as other paramyxoviruses. While we have confirmed that M proteins were conserved among individual viral species, any changes that are observed should be given attention and further researched.

\section{Acknowledgements}

This work was supported in part by the Croatian Science Foundation under the project number 6255 (to D.F.) and in part by the grant "Strengthening the capacity of CerVirVac for research in virus immunology and vaccinology", KK.01.1.1.01.0006, awarded to the Scientific Centre of Excellence for Virus Immunology and Vaccines and co-financed by the European Regional Development Fund.

\section{Disclosure Statement}

The authors declare that they have no conflicts of interest.

\section{References}

1 Afonso CL, Amarasinghe GK, Banyai K, Bao Y, Basler CF, Bavari S, et al: Taxonomy of the order Mononegavirales: update 2016. Arch Virol 2016;161:2351-2360.

-2 McCarthy AJ, Goodman SJ: Reassessing conflicting evolutionary histories of the Paramyxoviridae and the origins of respiroviruses with Bayesian multigene phylogenies. Infect Genet Evol 2010;10:97-107.

-3 Amarasinghe GK, Bào Y, Basler CF, Bavari S, Beer M, Bejerman N, et al: Taxonomy of the order Mononegavirales: update 2017. Arch Virol 2017;162:2493-2504.

4 van Regenmortel MHV, Burke DS, Calisher $\mathrm{CH}$, Dietzgen RG, Fauquet CM, Ghabrial SA, et al: A proposal to change existing virus species names to non-Latinized binomials. Arch Virol 2010;155:1909-1919.

5 Harrison MS, Sakaguchi T, Schmitt AP: Paramyxovirus assembly and budding: building particles that transmit infections. Int J Biochem Cell Biol 2010;42:1416-1429.

6 Schmitt AP, He B, Lamb RA: Involvement of the cytoplasmic domain of the hemagglutinin-neuraminidase protein in assembly of the paramyxovirus simian virus 5. J Virol 1999; 73:8703-8712.
7 Waning DL, Schmitt AP, Leser GP, Lamb RA: Roles for the cytoplasmic tails of the fusion and hemagglutinin-neuraminidase proteins in budding of the paramyxovirus simian virus 5. J Virol 2002;76:9284-9297.

8 Moll M, Klenk H-D, Maisner A: Importance of the cytoplasmic tails of the measles virus glycoproteins for fusogenic activity and the generation of recombinant measles viruses. J Virol 2002;76:7174-7186.

-9 Ghildyal R, Li D, Peroulis I, Shields B, Bardin PG, Teng MN, et al: Interaction between the respiratory syncytial virus $\mathrm{G}$ glycoprotein cytoplasmic domain and the matrix protein. J Gen Virol 2005;86:1879-1884.

10 Iwasaki M, Takeda M, Shirogane Y, Nakatsu Y, Nakamura T, Yanagi Y: The matrix protein of measles virus regulates viral RNA synthesis and assembly by interacting with the nucleocapsid protein. J Virol 2009;83:10374-10383.

11 Ray G, Schmitt PT, Schmitt AP: C-terminal DxD-containing sequences within paramyxovirus nucleocapsid proteins determine matrix protein compatibility and can direct foreign proteins into building particles. J Virol 2016;90:3650-3660.
12 Runkler N, Pohl C, Schneider-Schaulies S, Klenk HD, Maisner A: Measles virus nucleocapsid transport to the plasma membrane requires stable expression and surface accumulation of the viral matrix protein. Cell Microbiol 2007;9:1203-1214.

13 Patterson JB, Cornu TI, Redwine J, Dales S, Lewicki H, Holz a, et al: Evidence that the hypermutated $\mathrm{M}$ protein of a subacute sclerosing panencephalitis measles virus actively contributes to the chronic progressive CNS disease. Virology 2001;291:215-225.

14 Cathomen T, Mrkic B, Spehner D, Drillien R, Naef R, Pavlovic J, et al: A matrix-less measles virus is infectious and elicits extensive cell fusion: consequences for propagation in the brain. ЕMBO J 1998;17:3899-3908.

-15 Inoue M, Tokusumi Y, Ban H, Kanaya T, Shirakura M, Tokusumi T, et al: A new Sendai virus vector deficient in the matrix gene does not form virus particles and shows extensive cellto-cell spreading. J Virol 2003;77:6419-6429.

16 Coronel EC, Murti KG, Takimoto T, Portner A: Human parainfluenza virus type 1 matrix and nucleoprotein genes transiently expressed in mammalian cells induce the release of virus-like particles containing nucleocapsidlike structures. J Virol 1999;73:7035-7038. 
17 Takimoto T, Murti KG, Bousse T, Scroggs RA, Portner A: Role of matrix and fusion proteins in budding of Sendai virus. J Virol 2001; 75:11384-11391.

-18 Pohl C, Duprex WP, Krohne G, Rima BK, Schneider-Schaulies S: Measles virus M and F proteins associate with detergent-resistant membrane fractions and promote formation of virus-like particles. J Gen Virol 2007;88: 1243-1250.

19 Li M, Schmitt PT, Li Z, McCrory TS, He B, Schmitt AP: Mumps virus matrix, fusion, and nucleocapsid proteins cooperate for efficient production of virus-like particles. J Virol 2009;83:7261-7272.

-20 Sugahara F, Uchiyama T, Watanabe H, Shimazu Y, Kuwayama M, Fujii Y, et al: Paramyxovirus Sendai virus-like particle formation by expression of multiple viral proteins and acceleration of its release by $\mathrm{C}$ protein. Virology 2004;325:1-10.

-21 Leyrat C, Renner M, Harlos K, Huiskonen JT, Grimes JM: Structure and self-assembly of the calcium binding matrix protein of human metapneumovirus. Structure 2014;22:136148.

22 Latiff K, Meanger J, Mills J, Ghildyal R: Sequence and structure relatedness of matrix protein of human respiratory syncytial virus with matrix proteins of other negative-sense RNA viruses. Clin Microbiol Infect 2004;10: 945-948.

23 Kosakovsky Pond SL, Frost SDW: Not so different after all: a comparison of methods for detecting amino acid sites under selection. Mol Biol Evol 2005;22:1208-1222.

24 Darriba D, Taboada GL, Doallo R, Posada D: jModelTest 2: more models, new heuristics and parallel computing. Nat Methods 2012;9: 772 .

25 Karron RA, Collins PL: Parainfluenza viruses; in Knipe DM, Howley PM (ed): Fields Virology, ed 6. Philadelphia, Lippincott, Williams \& Wilkins, 2013, p 3177.

26 Jagušić M, Slovic A, Sternak S, MlinarićGalinović G, Forčić D: Genetic diversity of human metapneumovirus in hospitalized children with acute respiratory infections in Croatia. J Med Virol 2017;89:1885-1893.

27 Chomczynski P, Sacchi N: Single-step method of RNA isolation by acid guanidinium thiocyanate-phenol-chloroform extraction. Anal Biochem 1987;162:156-159.

28 Tamura K, Stecher G, Peterson D, Filipski A, Kumar S: MEGA6: molecular evolutionary genetics analysis version 6.0. Mol Biol Evol 2013;30:2725-2729.

-29 Zlateva KT, Lemey P, Moes E, Vandamme AM, Van Ranst M: Genetic variability and molecular evolution of the human respiratory syncytial virus subgroup $B$ attachment $G$ protein. J Virol 2005;79:9157-9167.
30 Zlateva KT, Lemey P, Vandamme A, van Ranst M: Molecular evolution and circulation patterns of human respiratory syncytial virus subgroup A: positively selected sites in the attachment G glycoprotein. J Virol 2004;78: 4675-4683.

31 Trento A, Casas I, Calderón A, Garcia-Garcia ML, Calvo C, Perez-Breña P, et al: Ten years of global evolution of the human respiratory syncytial virus BA genotype with a 60-nucleotide duplication in the $\mathrm{G}$ protein gene. J Virol 2010;84:7500-7512.

32 Agapov E, Sumino KC, Gaudreault-Keener M, Storch GA, Holtzman MJ: Genetic variability of human metapneumovirus infection: evidence of a shift in viral genotype without a change in illness. J Infect Dis 2006;193:396403.

33 Ghildyal R, Ho A, Jans DA: Central role of the respiratory syncytial virus matrix protein in infection. FEMS Microbiol Rev 2006;30:692705.

34 Battisti AJ, Meng G, Winkler DC, McGinnes LW, Plevka P, Steven AC, et al: Structure and assembly of a paramyxovirus matrix protein. Proc Natl Acad Sci USA 2012;109:139964000.

35 Zhang G, Zhang S, Ding B, Yang X, Chen L, Yan Q, et al: A leucine residue in the $C$ terminus of human parainfluenza virus type 3 matrix protein is essential for efficient virus-like particle and virion release. J Virol 2014;88: 13173-13188.

36 El Najjar F, Schmitt AP, Dutch RE: Paramyxovirus glycoprotein incorporation, assembly and budding: a three way dance for infectious particle production. Viruses 2014; 6:3019-3054.

37 Ciancanelli MJ, Basler CF: Mutation of YMYL in the Nipah virus matrix protein abrogates budding and alters subcellular localization. J Virol 2006;80:12070-12078.

38 Patch JR, Han Z, McCarthy SE, Yan L, Wang LF, Harty RN, et al: The YPLGVG sequence of the Nipah virus matrix protein is required for budding. Virol J 2008;5:137.

-39 Irie T, Shimazu Y, Yoshida T, Sakaguchi T: The YLDL sequence within Sendai virus M protein is critical for budding of virus-like particles and interacts with Alix/AIP1 independently of C protein. J Virol 2007;81:22632273.

-40 Košutić-Gulija T, Slovic A, Ljubin-Sternak S, Mlinarić-Galinović G, Forčić D: Genetic analysis of human parainfluenza virus type $3 \mathrm{ob}-$ tained in Croatia, 2011-2015. J Med Microbiol 2017;66:502-510.

41 Godoy C, Peremiquel-Trillas P, Andrés C, Gimferrer L, Uriona SM, Codina MG, et al: A molecular epidemiological study of human parainfluenza virus type 3 at a tertiary university hospital during 2013-2015 in Catalonia, Spain. Diagn Microbiol Infect Dis 2016;86: 153-159.
42 Almajhdi FN: Hemagglutinin-neuraminidase gene sequence-based reclassification of human parainfluenza virus 3 variants. Intervirology 2015;58:35-40.

43 Stone R, Takimoto T: Critical role of the fusion protein cytoplasmic tail sequence in parainfluenza virus assembly. PLoS One 2013;8:e61281.

44 Slovic A, Ivancic-Jelecki J, Ljubin-Sternak S, Galinović GM, Forcic D: A molecular epidemiological study of human respiratory syncytial virus in Croatia, 2011-2014. Infect Genet Evol 2016;44:76-84.

45 Watkinson RE, Lee B: Nipah virus matrix protein: expert hacker of cellular machines. FEBS Lett 2016;590:2494-2511.

46 Sabo Y, Ehrlich M, Bacharach E: The conserved YAGL motif in human metapneumovirus is required for higher-order cellular assemblies of the matrix protein and for virion production. J Virol 2011;85:6594-6609.

-47 Li X, Guo L, Kong M, Su X, Yang D, Zou M, et al: Design and evaluation of a multi-epitope peptide of human metapneumovirus. Intervirology 2016;58:403-412.

48 Cardin RD, Brooks JW, Sarawar SR, Doherty PC: Progressive loss of CD8+ T cell-mediated control of a gamma-herpesvirus in the absence of CD4+ T cells. J Exp Med 1996;184:863-871.

49 Chow WZ, Chan YF, Oong XY, Ng LJ, Nor'E SS, Ng KT, et al: Genetic diversity, seasonality and transmission network of human metapneumovirus: identification of a unique sublineage of the fusion and attachment genes. Sci Rep 2016;6:27730.

50 Cane PA, Pringle CR: Evolution of subgroup A respiratory syncytial virus: evidence for progressive accumulation of amino acid changes in the attachment protein. J Virol 1995;69:2918-2925.

51 Noton SL, Fearns R: Initiation and regulation of paramyxovirus transcription and replication. Virology 2015;479-480:545-554.

52 Moudy RM, Sullender WM, Wertz GW: Variations in intergenic region sequences of $\mathrm{Hu}$ man respiratory syncytial virus clinical isolates: analysis of effects on transcriptional regulation. Virology 2004;327:121-133.

53 Cartee TL, Megaw AG, Oomens AGP, Wertz GW: Identification of a single amino acid change in the human respiratory syncytial virus $L$ protein that affects transcriptional termination. J Virol 2003;77:7352-7360.

54 Piyaratna R, Tollefson SJ, Williams JV: Genomic analysis of four human metapneumovirus prototypes. Virus Res 2011;160:200-205.

55 Lingemann M, Surman S, Amaro-Carambot E, Schaap-Nutt A, Collins PL, Munir S: The aberrant gene-end transcription signal of the matrix $\mathrm{M}$ gene of human parainfluenza virus type 3 downregulates fusion F protein expression and the F-specific antibody response in vivo. J Virol 2015;89:3318-3331.
Analysis of Matrix Proteins in Families Paramyxoviridae and Pneumoviridae
Intervirology

DOI: $10.1159 / 000487049$ 